\title{
Influence of Titanium Dioxide Preparation Method on Photocatalytic Degradation of Organic Dyes
}

\author{
Vanja Gilja, ${ }^{1}$ Ljerka Kratofil Krehula, ${ }^{1, *}$ Zvonimir Katančić, ${ }^{1}$ Stjepko Krehula, ${ }^{2}$ Zlata Hrnjak-Murgić, ${ }^{1, \# ~ J a d r a n k a ~ T r a v a s-S e j d i c ~}{ }^{3,4}$ \\ 1 Faculty of Chemical Engineering and Technology, University of Zagreb, Marulićev trg 19, 10000 Zagreb, Croatia \\ 2 Ruđer Bošković Institute, Bijenička cesta 54, 10000 Zagreb, Croatia \\ 3 Polymer Electronics Research Centre, School of Chemical Sciences, University of Auckland, Auckland, New Zealand \\ 4 MacDiarmid Institute for Advanced Materials and Nanotechnology, Victoria University of Wellington, Wellington, New Zealand \\ * Corresponding author's e-mail address: krehula@fkit.hr \\ \# Corresponding author's e-mail address: zhrnjak@fkit.hr
}

RECEIVED: January 23, 2018 * REVISED: June 15, 2018 * ACCEPTED: June 18, 2018

Abstract: Titanium catalysts $\left(\mathrm{TiO}_{2}\right)$ were synthesized by three different methods. Their photocatalytic activity was validated through photodegradation of Reactive Red 45 (RR45) azo dye and Acid Blue 25 (AB25) anthraquinone dye in an aqueous solution under UV irradiation. $\mathrm{TiO}_{2}$ photocatalysts were characterized by FTIR, XRD and SEM. Photosensitivity and $\mathrm{TiO}_{2}$ activity range were characterized by UV/Vis spectroscopy. Photocatalytic validation has been made by way of determining the degree of RR45 and AB25 removal. TOC was determined as a measure of the mineralization of RR45 and $\mathrm{AB} 25$ by photocatalysis. The stability of $\mathrm{TiO}_{2}$ catalysts and a possibility of using them in consecutive photocatalysis cycles have also been studied. The results show that the photocatalytic efficiency depends on the crystal structure of $\mathrm{TiO}_{2}$. The size of crystallites depends on synthesis conditions. From the results of photocatalytic efficiency it is concluded that the chemical interaction between a catalyst and a dye strongly depends on the dye chemical structure.

Keywords: titanium dioxide, photocatalysis, organic dyes, wastewater treatment.

\section{INTRODUCTION}

$\mathbf{T}$ HE application of advanced oxidation processes (AOPs) to wastewater treatment is particularly useful because AOPs are environmentally friendly, cost-effective and lead to a complete degradation (mineralization) of organic pollutants. In other words, there is no need for further purification or disposal of pollutants as in other water treatment processes (mechanical and physical/mechanical). Primary intention is to reduce the concentration of organic carbon in wastewater at the lowest possible treatment costs. ${ }^{[1-3]}$ Heterogeneous photocatalysis is one of AOPs that removes organic pollutants from an aqueous medium using metal oxides, i.e., semiconductor catalysts $\left(\mathrm{TiO}_{2}, \mathrm{ZnO}, \mathrm{Fe}_{2} \mathrm{O}_{3}\right)$ in combination with UV or visible light irradiation. ${ }^{[4,5]}$ Photocatalytic reactions have been studied by various research groups: by Matsuoka, Anpo, Horiuchi ${ }^{[6-9]}$ and by Mori, Yamashita et al. ${ }^{[10-12]}$ as well as some other researchers. ${ }^{[13-17]}$ Knowledge about the bulk properties of $\mathrm{TiO}_{2}$ with different crystal structures has been comprehensively reviewed by Diebold. ${ }^{[18]}$ Among $\mathrm{TiO}_{2}$ crystalline forms rutile is the most stable phase in bulk form, whereas two other phases, anatase and brookite are metastable. Upon heating both anatase and brookite can transform irreversibly into rutile. The phase transition of $\mathrm{TiO}_{2}$ anatase nanoparticles to $\mathrm{TiO}_{2}$ rutile nanoparticles starts from the interfaces between the agglomerated anatase crystallites, leading to a bulk phase transition. ${ }^{[19-21]}$ The process of anatase and brookite transition to the rutile phase strongly depends on the $\mathrm{TiO}_{2}$ particle size. The most stable phase of $\mathrm{TiO}_{2}$ in the temperature range between $325^{\circ} \mathrm{C}$ and $750{ }^{\circ} \mathrm{C}$ is rutile with particle size above $35 \mathrm{~nm}$, while anatase is the most stable phase with particle size under $11 \mathrm{~nm}$, and brookite phase between 11 and $35 \mathrm{~nm} \cdot{ }^{[1]} \mathrm{A}$ larger band gap causes anatase to exhibit photocatalytic activity different from that of rutile. Anatase $\mathrm{TiO}_{2}$ is the most studied photocatalyst and its surface area is usually larger than that of rutile, resulting in an enhanced adsorption capability and more active sites. Anatase can 
generate more efficient charge separation due to the existence of more oxygen vacancies. Owing to such advantages it usually shows a much higher photocatalytic activity than rutile under UVA irradiation. ${ }^{[22]}$ Rutile has the band gap value smaller than that of anatase. Both pure phases absorb light in the UV region. Due to the presence of some defects or dopants, $\mathrm{TiO}_{2}$ samples start absorbing light under solar irradiation. Thus the photocatalytic activity is controlled by the microstructure (crystalline phase, crystallinity and crystallite size) and by the surface area (particle size).[23,24] The former affects surface properties and the recombination rate of the photogenerated electrons and holes, while the latter contributes to an efficient adsorption of the pollutant. The photocatalytic reaction can be divided into the reduction reaction and the oxidation reaction. The reduction reaction can be initiated by photoexcited electrons in the conductive band (CB) with electron acceptors. The reduction capability depends on the position of a $\mathrm{CB}$ minimum (CBM). The oxidation reaction is driven by holes in the valence band (VB). The position of a VB maximum (VBM) sets the oxidation capability, Figure 1.(a). The semiconductor position of a photocatalyst CBM needs to be located at a more negative potential than the redox potential of $\mathrm{H}^{+} / \mathrm{H}_{2}(\mathrm{pH} 7)$ to obtain hydrogen from the reaction of water splitting. The VBM must be situated at a more positive potential than the redox potential of $\mathrm{O}_{2} / \mathrm{H}_{2} \mathrm{O}$ $(\mathrm{pH} 7)$. The energy for generation of electron-hole pairs in the catalyst needs to be greater than the energy gap $\left(E_{\mathrm{g}}\right)$. The creation of electron $\left(\mathrm{e}^{-}\right)$-hole $\left(\mathrm{h}^{+}\right)$pairs can be induced by photon absorption and involves several different processes. Three of them are the most important: (a) electronhole pairs are separated and successfully migrate to the $\mathrm{TiO}_{2}$ surface, then to some adsorbed species or to the solvent; (b) separated charge carriers are captured by the defect sites in bulk and/or on the surface region of $\mathrm{TiO}_{2}$; (c) separated charge carriers recombine and then release energy in bulk or on the $\mathrm{TiO}_{2}$ surface, Figure 1. ${ }^{[24]}$ The processes described in Figure 1.(b) and (c) are known as deexcitation processes because the formed charges do not perform a photocatalytic reaction since only the charges that are transferred to the reactants via the $\mathrm{TiO}_{2}$ surface could contribute to photocatalytic reactions. It should be pointed out that the defect sites in bulk or on the surface of $\mathrm{TiO}_{2}$ may serve as recombination centers and thus decrease the photocatalytic reaction efficiency. ${ }^{[25]}$ To achieve a specific morphology and high surface it is important to control the crystallinity, porosity and composition of the $\mathrm{TiO}_{2}$ catalyst. ${ }^{[26,27]}$ Therefore the physical/chemical properties of a catalyst may be quite different, depending on their phase structure and particle size.

The goal of this research was to establish how the photocatalytic activity of a $\mathrm{TiO}_{2}$ catalyst had been affected by synthesis conditions including the starting chemicals,

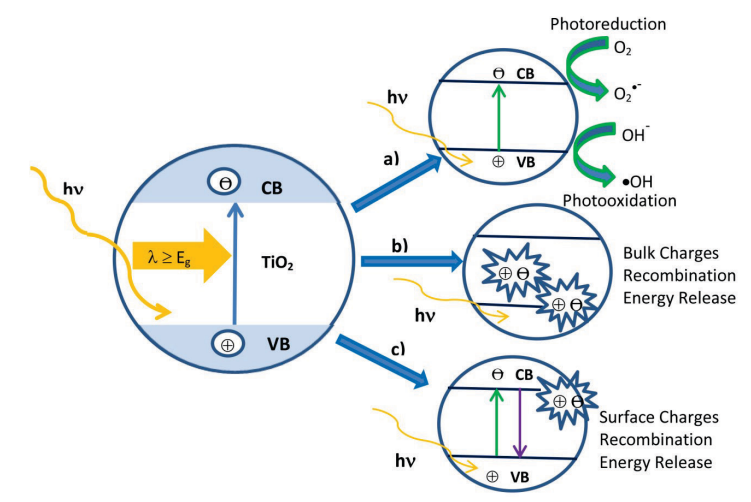

Figure 1. The most important processes in the surface photocatalysis: a) electron-hole pairs are separated and successfully migrate to the surface of $\mathrm{TiO}_{2}$ and then to adsorbed organic or inorganic species or to the solvent; (b) separated charge carriers are captured by the defect sites in bulk and/or on the surface region of $\mathrm{TiO}_{2}$; (c) separated charge carriers recombine and release the energy in the form of heat or photon in bulk and/or on the surface region of $\mathrm{TiO}_{2}$.

reaction duration and temperature as well as the duration of calcination. The objective was also to establish $\mathrm{TiO}_{2}$ photodegradation efficiency in the removal of Reactive Red 45 (RR45) or Acid Blue 25 (AB25) dyes from wastewater.

\section{EXPERIMENTAL}

\section{Materials}

Hydrochloric acid $(\mathrm{HCl}, 37 \%)$, acetic acid $\left(\mathrm{C}_{2} \mathrm{H}_{4} \mathrm{O}_{2}, 99.8 \%\right)$ and anhydrous ethanol $\left(\mathrm{C}_{2} \mathrm{H}_{5} \mathrm{OH}, 99.7 \%\right)$ were all purchased from Kemika Zagreb. All the chemicals were used without any further purification. Tetra- $n$-butyl titanate (TBT) $\left(\mathrm{Ti}_{(}\left(\mathrm{OC}_{4} \mathrm{H}_{9}\right)_{4}, 99 \%\right)$ was purchased from Acros Organics. Didecyl dimethyl ammonium chloride (DDAC) $\left(\mathrm{C}_{22} \mathrm{H}_{48} \mathrm{CIN}\right)$ was supplied from Lonza $L t d$. Commercial organic dyes C.I. Reactive Red 45 (RR45) and C.I. Acid Blue 25 (AB25) from CibaGeigy, Basel, were used without any purification. All aqueous solutions were prepared with deionized water that had been passed through a Merck Millipore Direct-Q3 UV water purification system.

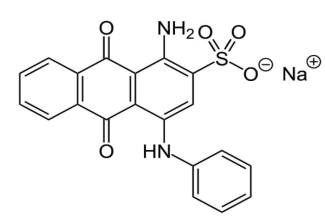

RR45

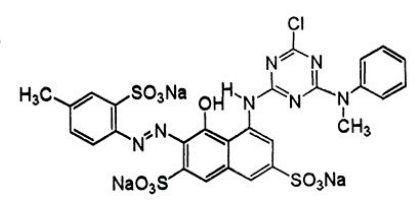

AB25 


\section{Synthesis of $\mathrm{TiO}_{2}$ Photocatalysts}

TiV photocatalyst was prepared by the sol-gel method using tetra-n-butyl titanate as a precursor.

Solution A was prepared by dissolving $17 \mathrm{~mL}$ of TBT in $30 \mathrm{~mL}$ anhydrous ethanol with stirring (300 rpm) for $30 \mathrm{~min}$. Another solution that contained $28.35 \mathrm{~mL}$ of anhydrous ethanol, $7.25 \mathrm{~mL}$ of deionized water and $20 \mathrm{~mL}$ of $3 \mathrm{M}$ acetic acid was slowly added to the above solution, with magnetic stirring at room temperature. The prepared mixture was further hydrolyzed for $60 \mathrm{~min}$ with stirring until a transparent sol was obtained, which was aged for $20 \mathrm{~h}$ at room temperature.

Thus prepared $\mathrm{TiO}_{2}$ was dried at $80^{\circ} \mathrm{C}$ in an oven for $12 \mathrm{~h}$ and calcined for $3 \mathrm{~h}$ at three different temperatures: $400{ }^{\circ} \mathrm{C}, 500{ }^{\circ} \mathrm{C}, 600^{\circ} \mathrm{C}$.

Synthesis of TiCh photocatalyst was made from $12.8 \mathrm{~mL}$ of TBT introduced dropwise into $50 \mathrm{~mL}$ of $3 \mathrm{M}$ acetic acid and stirred with a magnetic stirrer for $60 \mathrm{~min}$. The resultant solution was then transferred to an Erlenmeyer flask and heat-treated at $105{ }^{\circ} \mathrm{C}$ for $24 \mathrm{~h}$. Sample was washed with absolute ethanol for three times and dried in a vacuum oven at $100{ }^{\circ} \mathrm{C}$ for $12 \mathrm{~h}$. The obtained material was ground into powder and calcined for $3 \mathrm{~h}$ at the following temperatures: $400{ }^{\circ} \mathrm{C}, 500{ }^{\circ} \mathrm{C}$, $600^{\circ} \mathrm{C}$.

Synthesis of TiB photocatalyst was made by the sol-gel method. Solution A consisted of $20 \mathrm{~mL}$ of TBT dissolved in $20 \mathrm{~mL}$ of anhydrous ethanol. $20 \mathrm{ml}$ of $3 \mathrm{M}$ acetic acid was then added dropwise to the solution that was stirred for $15 \mathrm{~min}$. Solution B consisted of $2.1 \mathrm{~mL}$ of DDAC mixed with $60 \mathrm{~mL}$ of anhydrous ethanol and $20 \mathrm{~mL}$ of distilled water was stirred for $15 \mathrm{~min}$ at room temperature. Then solution $B$ was added dropwise to solution $A$. The resultant mixture was stirred for $45 \mathrm{~min}$ with a high speed disperser (7500 rpm), then transferred to an Erlenmeyer flask and heated at $85^{\circ} \mathrm{C}$ in an oven for $24 \mathrm{~h}$ until a transparent sol was obtained. The obtained sample was then washed several times with distilled water and dried at $100{ }^{\circ} \mathrm{C}$ for $12 \mathrm{~h}$. Finally the samples were calcined for $3 \mathrm{~h}$ at the following temperatures: 400 ${ }^{\circ} \mathrm{C}, 500{ }^{\circ} \mathrm{C}, 600{ }^{\circ} \mathrm{C}$.

\section{Characterization}

FTIR analysis was performed made with Perkin-Elmer Spectrum One FT-IR spectrometer in the range of 4000 $450 \mathrm{~cm}^{-1}$. Samples for characterization were prepared as pellets with $\mathrm{KBr}$.

UV/Vis spectroscopy measurements of synthesized $\mathrm{TiO}_{2}$ photocatalysts were made over the range from 200 to $800 \mathrm{~nm}$ at a spectral resolution of $\sim 0.3-10 \mathrm{~nm}$ using Ocean Optics USB 2000+ Spectrometer. Measured reflectance was converted according to the Kubelka-Munk equation 1 , where $\mathrm{R}$ is reflectance (\%),

$$
K=\frac{(1-R)^{2}}{2 R}
$$

$(K \times h v)^{1 / 2}$ plotted against $h v$ is known as a Tauc plot. The value associated with the point of intersection of the line tangent to the plotted curve inflection point with horizontal axis ( $h v$ axis) is the band gap $\left(E_{\mathrm{g}}\right)$ value.

$$
(K \times h v)^{1 / 2}=f(h v)
$$

The microstructure and morphology of synthesized photocatalysts were studied by scanning electron microscope (SEM) Tescan Vega 3.

Samples were also characterized by X-ray powder diffractometer ItalStructures APD 2000

$\left(\lambda\left(\mathrm{Cu} K_{\alpha}\right)=1.5405 \AA\right)$ in the scan range $20^{\circ}-90^{\circ}$. The Rietveld refinement method was used for crystal structure analysis.

\section{Photocatalytic Activity Test}

The experiments were performed with waste water containing $30 \mathrm{mg} \mathrm{L}^{-1}$ of dyes (Reactive Red 45 and Acid Blue 25) and $1 \mathrm{~g} \mathrm{~L}^{-1}$ of synthesized photocatalyst (TiV, TiCh, TiB) in a glass waterjacketed batch reactor with an UVP Pen-Ray lamp (irradiation in the UVA region: 315 to $400 \mathrm{~nm}$ ) placed in the middle of the reactor. The characteristics of the UV lamp (Pen-Ray Lamp, 90-001901) used in photo experiments were: $365 \mathrm{~nm}$ longwave, 2.25-inches lighted length, lamp operating voltage 260 , lamp body temperature $\left({ }^{\circ} \mathrm{C}\right)$ 145.

In all the experiments the reaction system was stirred in the dark for $90 \mathrm{~min}$ to achieve the adsorption/desorption equilibrium before irradiation. At given irradiation time intervals an aliquot of about $3 \mathrm{~mL}$ was sampled and filtered through a $0.45 \mu \mathrm{m}$ membrane filter to remove the remaining particles. The filtrates were analyzed by recording variations of absorption in the UV-Vis spectra of RR45 and AB25 dyes at a wavelength of $542 \mathrm{~nm}$ and 622 $\mathrm{nm}$, respectively, using a Perkin Elmer Lambda EZ 201 spectrophotometer. Photodegradation efficiency was estimated by determining the concentration $(C)$ of dyes after a certain irradiation time where $C_{0}$ was used as the initial value before irradiation. The extent of RR45 and AB25 mineralization was determined on the basis of the total organic carbon (TOC) content; measurements were taken using a TOC analyzer, TOC-VCPN 5000 A, Shimadzu.

\section{Consecutive Cycles of Photocatalysis}

Multiple experiments involving photocatalysis were carried out under identical photocatalytic conditions to monitor the efficiency of the "recycled" photocatalyst. After each 
cycle of photocatalysis samples were allocated by decantation, then dried at room temperature for $12 \mathrm{~h}$ and reused in the next cycle. The efficiency of the photocatalyst over four cycles of photodegradation is given as a percentage of degraded RR45 and AB25 calculated according to the equation. Degradation is given as $(\%)=$ $\left(A_{0}-A\right) / A_{0} \times 100 \%$, where $A_{0}$ and $A$ were the initial and the reacting absorbance, respectively.

\section{RESULTS AND DISCUSSION}

\section{FTIR Spectroscopy}

FTIR spectra of the variously synthesized $\mathrm{TiO}_{2}$ nanocrystals calcined at $400{ }^{\circ} \mathrm{C}, 500{ }^{\circ} \mathrm{C}$ and $600^{\circ} \mathrm{C}$ are shown in Figure 2 . The broad adsorption band at $3428 \mathrm{~cm}^{-1}$ and the band at $1624 \mathrm{~cm}^{-1}$ are assigned to the stretching and bending modes characteristic of physisorbed water on oxide supports. ${ }^{[28]}$ This is observed for all samples regardless of the phase composition, morphology and aggregation, which can play a critical role in the specific application.

Very small bands at $2923 \mathrm{~cm}^{-1}, 2846 \mathrm{~cm}^{-1}$ and $1382 \mathrm{~cm}^{-1}$ corresponding to the $\mathrm{C}-\mathrm{H}$ vibrations of the $-\mathrm{CH}_{3}$ groups in the hydrocarbon moiety are assumed to originate from the acetic acid used in synthesis (higher absorbance is seen for TiCh and TiV samples). The band at $2350 \mathrm{~cm}^{-1}$ originates from $\mathrm{CO}_{2}$ in the air. ${ }^{[29]}$ Broad absorption bands that appear in the low frequency region of the infrared spectrum located in the range $450-850 \mathrm{~cm}^{-1}$ are characteristic of a Ti-O-Ti symmetric stretching vibration mode ${ }^{[4]}$ with the observed maximum at around $500 \mathrm{~cm}^{-1}$ corresponding to the Ti-O stretching vibration formed during the condensation reaction of sol-gel synthesis. The change in absorbance intensity at higher calcination temperatures indicates the change in crystallinity, less welldefined anatase crystals. ${ }^{[5]}$ TiV 500 and TiV 600 are the only studied $\mathrm{TiO}_{2}$ samples containing rutile, which is confirmed by XRD. This was also confirmed by FTIR results where a peak at $490-510 \mathrm{~cm}^{-1}$ is present indicating the rutile phase. At the temperature increased from 500 to $600{ }^{\circ} \mathrm{C}$ there was a slight shift of this peak and an increase in its intensity. ${ }^{[30]}$

From the FTIR spectra change in vibration bands it can be concluded that the synthesis conditions (temperature, duration), various starting chemicals and calcination temperature affect the $\mathrm{TiO}_{2}$ chemical composition and structure. The presence of some groups even in very low concentrations can affect interactions between the catalyst and pollutant due to the variously charged surface of the $\mathrm{TiO}_{2}$ catalyst.

\section{X-ray Diffraction}

The X-ray powder diffraction patterns of synthesized $\mathrm{TiO}_{2}$ samples (Figure 3 ) revealed the presence of the anatase
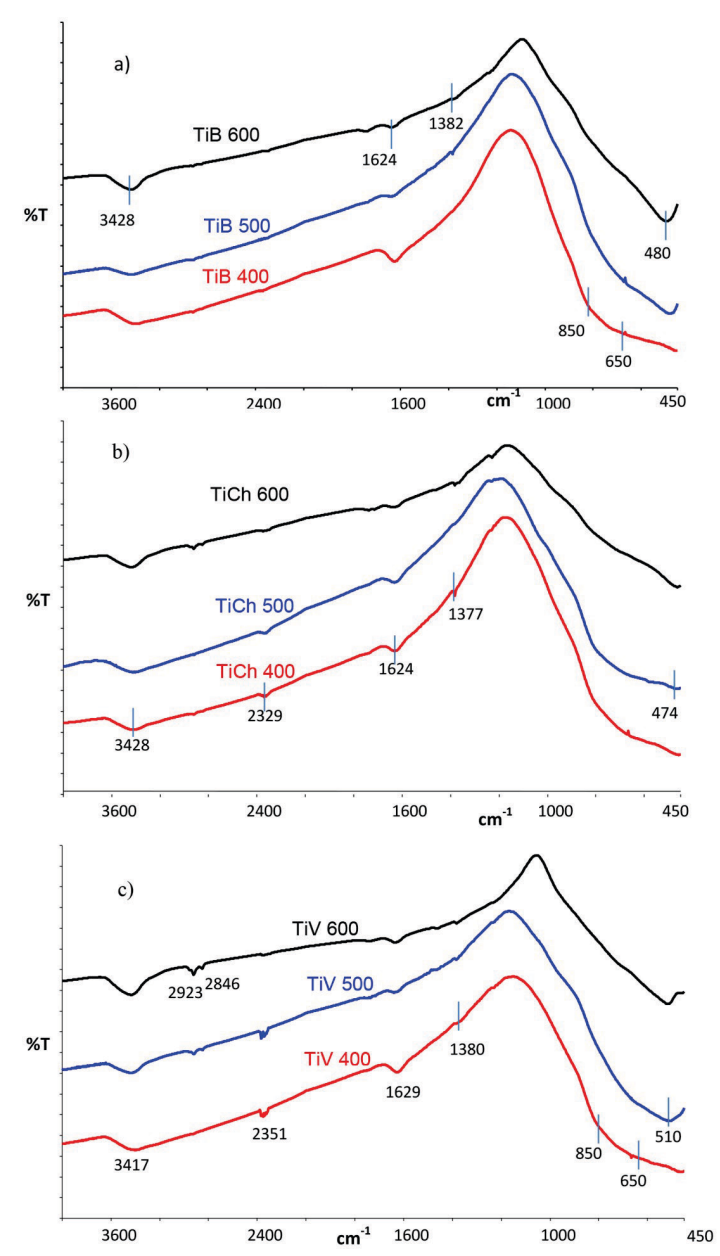

Figure 2. FTIR spectra of synthesized $\mathrm{TiO}_{2}$; a) $\left.\mathrm{TiB}, \mathrm{b}\right) \mathrm{TiCh}$, c) TiV calcined at $400{ }^{\circ} \mathrm{C}$ (red), $500{ }^{\circ} \mathrm{C}$ (blue) and $600{ }^{\circ} \mathrm{C}$ (black).

$\mathrm{TiO}_{2}$ phase as a single phase in all samples except in samples TiV 500 and TiV 600 where rutile $\mathrm{TiO}_{2}$ was also present. The Rietveld refinement was used for crystal structure analysis. The majority of synthesized $\mathrm{TiO}_{2}$ samples were pure anatase, only two samples contained rutile: TiV 500 (91.5\% anatase and $8.5 \%$ rutile) and TiV 600 ( $56 \%$ anatase and $44 \%$ rutile). The size of anatase $\mathrm{TiO}_{2}$ crystallites (Table 1) was estimated from the line width of the strongest anatase XRD line (101) using the Scherrer equation. ${ }^{[31]}$ From these results it can be concluded that the synthesis method has a significant impact on the size of crystallites. Furthermore, a higher calcination temperature induced an increase in the anatase $\mathrm{TiO}_{2}$ crystallite size. The Rietveld refinement method showed that $\mathrm{P} 25$ consisted of $87.6 \%$ anatase and $12.4 \%$ rutile. The size of $\mathrm{TiO}_{2}$ crystallites was determined by the Scherrer equation to the value of $24 \mathrm{~nm}$. 
Table 1. The size of anatase $\mathrm{TiO}_{2}$ crystallites estimated from the line width of the strongest anatase XRD line (101) by using the Scherrer equation. ${ }^{[18]}$

\begin{tabular}{ccccc}
\hline $\mathrm{TiO}_{2}$ samples & & & \\
\cline { 1 - 3 } Calcined temperature & $\mathrm{TiB} / \mathrm{nm}$ & $\mathrm{TiCh} / \mathrm{nm}$ & $\mathrm{TiV} / \mathrm{nm}$ \\
\hline $400^{\circ} \mathrm{C}$ & 11 & 14 & 18 \\
$500^{\circ} \mathrm{C}$ & 28 & 18 & 43 \\
$600{ }^{\circ} \mathrm{C}$ & 41 & 34 & 51 \\
\hline
\end{tabular}

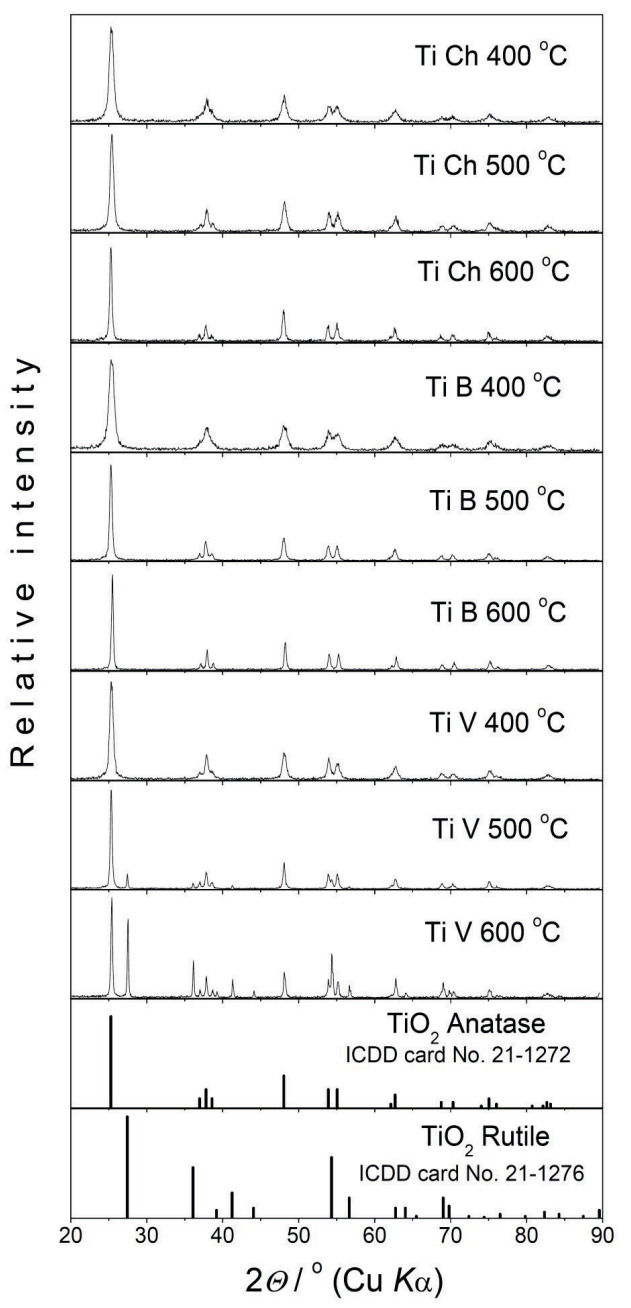

Figure 3. XRD difractogram of synthesized $\mathrm{TiO}_{2}$; a) $\mathrm{TiCh}$ b) TiB, c) TiV calcined at $400{ }^{\circ} \mathrm{C}, 500{ }^{\circ} \mathrm{C}, 600^{\circ} \mathrm{C}$.

\section{UV/Vis Spectroscopy}

The reflectance/absorbance capacity of the synthesized $\mathrm{TiO}_{2}$ photocatalyst was recorded by UV/Vis spectroscopy, Figure 4. a), b), c). For all studied $\mathrm{TiO}_{2}$ photocatalysts the spectrum consisted of single reflectance below ca. $400 \mathrm{~nm}$, which is attributed to the charge-transfer from the valence band (mainly formed by $2 p$ orbitals of the oxide cations) to the conduction band (mainly formed by $3 \mathrm{~d} \mathrm{t}_{2 \mathrm{~g}}$ orbitals of the $\mathrm{Ti}^{4+}$ cations). ${ }^{[22]}$ In this region there is some deviation in reflectance since it was shifted to higher visible wavelengths for TiB600, TiCh400 and TiV600 samples. These results indicate that the valence band electrons of $\mathrm{TiO}_{2}$ were excited at a wavelength longer than $390 \mathrm{~nm}$ (beyond UVA irradiation), suggesting that the phase composition (anatase and rutile) was changed. Since XRD analysis indicated the rutile phase only for TiV500 and TiV600 samples, the difference in reflectance is also attributed to variations in crystals and crystallite size. According to a previously published report, ${ }^{[17]}$ higher calcination temperature contributes to the formation of a rutile phase that results in the formation of larger size crystals. The difference in reflectance in the region from $420 \mathrm{~nm}$ to $800 \mathrm{~nm}$ wavelength (linear part of the curves) of the synthesized $\mathrm{TiO}_{2}$ photocatalyst suggests that the crystals formed in samples were not of equal size due to aggregation after calcination. Some $\mathrm{TiO}_{2}$ samples were slightly coloured (slightly grey or yellowish), which indicates the presence of by-products (as a result of chemicals used in synthesis). Such coloured samples absorb in the visible region. From the XRD results in Table 1 it is observed that synthesis conditions as well as calcination temperatures affected the size of crystallites. Figure $4 \mathrm{~d}$ ) shows the reflectance $(\%)=f$ (wavelength) relationship prepared $\mathrm{TiO}_{2}$ samples. The value associated with the point of intersection of the line tangent to the plotted curve inflection point with the horizontal axis (hv axis) is the band gap (Eg) value. Values of the band gap for studied samples are listed in Table 2. Differences among the band gaps are attributed to variations in synthesis conditions, the crystalline size and the content of the impurities.

Table 2. Indirect band gap (Eg) transitions for synthesized $\mathrm{TiO}_{2}$ samples.

\begin{tabular}{cc}
\hline Samples & $E_{\mathrm{g}} / \mathrm{eV}$ \\
\hline TiB400 & 3.22 \\
TiB500 & 3.19 \\
TiB600 & 3.14 \\
TiCh400 & 3.26 \\
TiCh500 & 3.15 \\
TiCh600 & 3.15 \\
TiV400 & 3.19 \\
TiV500 & 3.24 \\
TiV600 & 2.98 \\
\hline
\end{tabular}



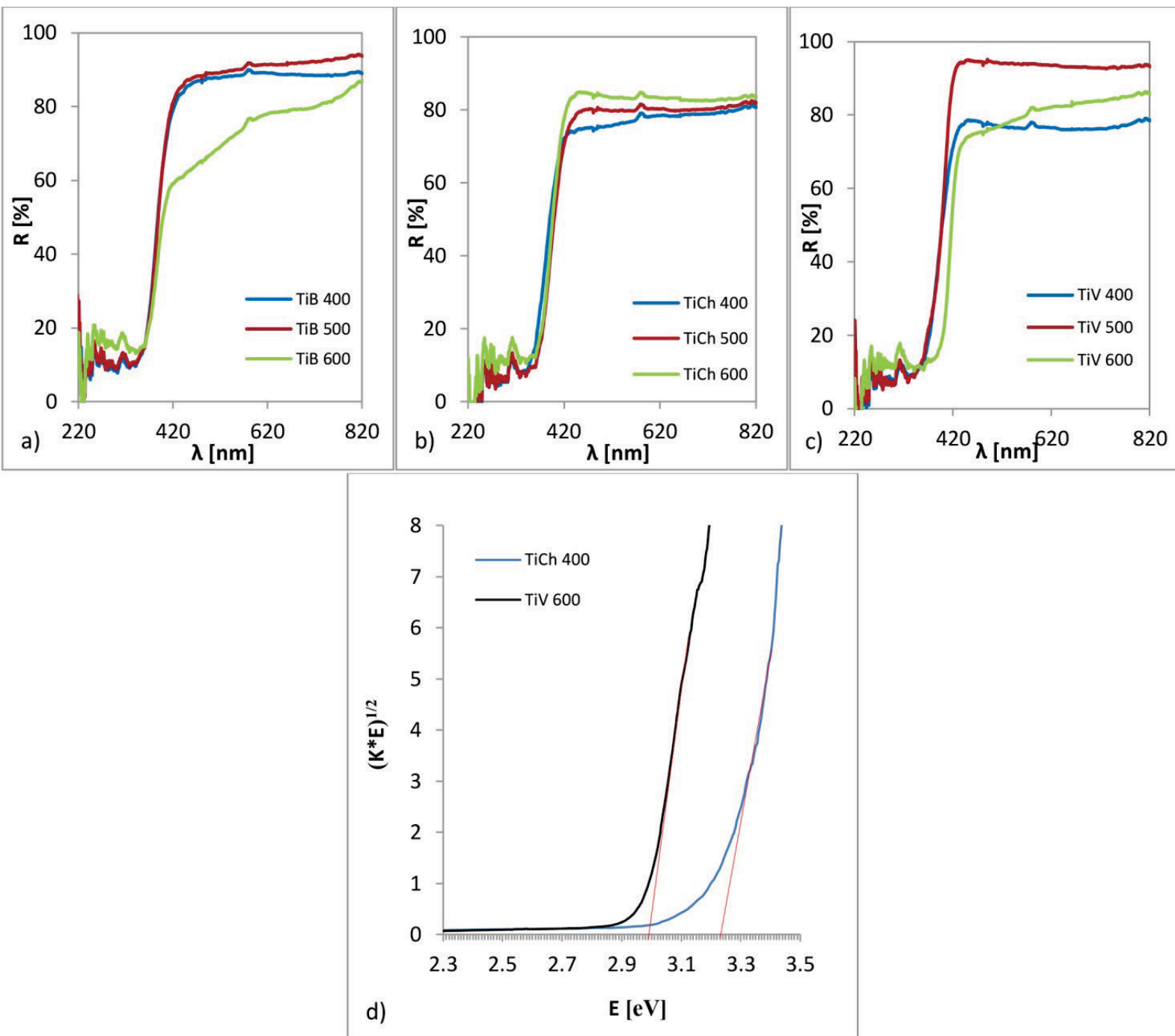

Figure 4. UV/Vis reflectance spectra of synthesized $\mathrm{TiO}_{2} ; \mathrm{a}$ ) TiB, b) TiCh, c) TiV calcined at various temperatures: $400{ }^{\circ} \mathrm{C}, 500{ }^{\circ} \mathrm{C}$, $\left.600{ }^{\circ} \mathrm{C}, \mathrm{d}\right)$ absorbance spectra for the indirect electronic transition $(K E)^{1 / 2} \mathrm{vs} E / \mathrm{eV}$ of synthesized $\mathrm{TiO}_{2}$ samples.

\section{SEM Analysis}

SEM micrographs and the morphology of the synthesized $\mathrm{TiO}_{2}$ photocatalyst are presented in Figure 5. It is evident that the morphology differs depending on the method of synthesis and calcination temperature. The finest morphology is observed in TiCh400 and TiV400 photocatalysts that changed significantly with an increase in calcination temperature. The average particle size increased from cca $0.2 \mu \mathrm{m}$ for calcination at $400{ }^{\circ} \mathrm{C}$ to 10 $15 \mu \mathrm{m}$ for calcination at $500^{\circ} \mathrm{C}$. For TiV sample the particle size increase was even more significant, from $0.3 \mu \mathrm{m}$ after calcination at $400^{\circ} \mathrm{C}$ to $15-40 \mu \mathrm{m}$ at $500^{\circ} \mathrm{C}$ and $600^{\circ} \mathrm{C}$ due to the rutile phase formed. The morphology of TiB sample appeared to be different, and mostly presents large and medium-sized particles with a small fraction of very small particles. The size of large and medium-sized particles is in the range of $5-20 \mu \mathrm{m}$, whereas very small particles measure less than one micron $(c c a 0.2 \mu \mathrm{m})$. To enhance the photocatalytic activity of the catalyst the microstructure needs to be controlled as well as the enlargement of its surface area.[32,19]

The former affects the surface property and influences the recombination rate of the photogenerated electrons and holes, while the latter contributes to reactant-efficient adsorption. The anatase $\mathrm{TiO}_{2}$, which possesses the highest photocatalytic activity among anatase, rutile and brookite, may change phase to rutile in a calcination process, ${ }^{[3,34]}$ accompanied by a significant surface area reduction (Fig. 5i)), resulting in a diminished photocatalytic activity in the UVA region. 


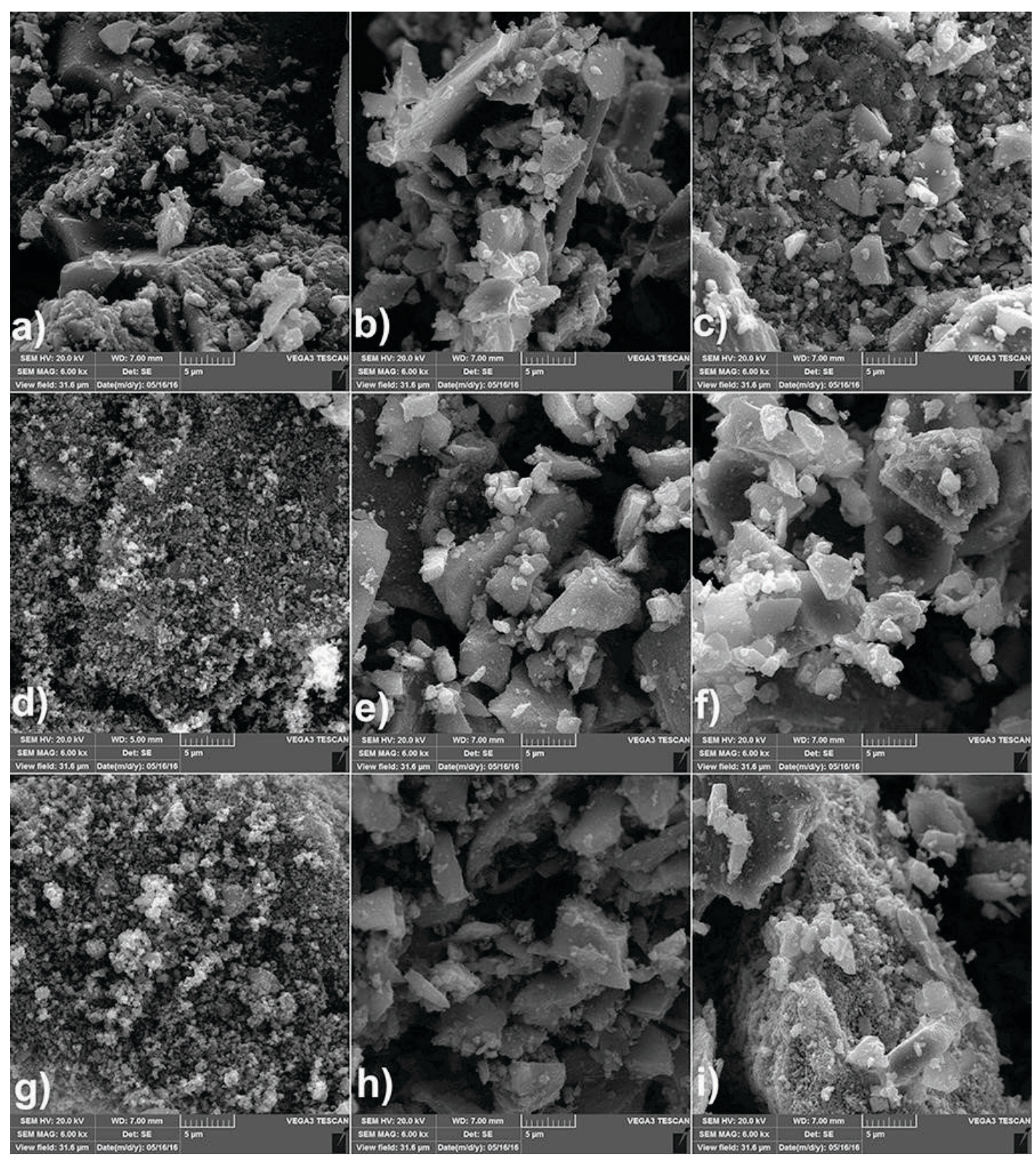

Figure 5. SEM micrographs of synthesized $\mathrm{TiO}_{2}$ calcined at 400, 500, $600{ }^{\circ} \mathrm{C}$, a) $\mathrm{TiB} 400$, b) $\mathrm{TiB} 500$, c) TiB600, d) $\mathrm{TiCh} 400$, e) TiCh500, f) TiCh600, g) TiV400, h) TiV500, i) TiV600.

\section{Photocatalytic Activity}

The photocatalytic activity of the synthesized $\mathrm{TiO}_{2}$ photocatalyst was evaluated through the degradation of $30 \mathrm{mg} \mathrm{L}^{-1}$ of RR45 and AB25 dyes in an aqueous solution with $1.0 \mathrm{mg}$ $\mathrm{L}^{-1}$ of catalyst and upon UV-A irradiation. The efficiency of the catalyst systems was investigated at $\mathrm{pH}$ that resulted from the presence of a dye and a photocatalyst: 6.2 in the case of RR45 and 5.5 in the case of AB25. The pH was not further adjusted. The decomposition ratio of dyes $\left(C / C_{0}\right)$ was correlated to the reaction time $(t)$ and the results are shown in Figures 6 and 9. In Figure 6 only small differences in the adsorption process for studied photocatalyst samples are seen and the maximum adsorption was reached at $5 \%$. On the other hand, the photocatalytic process efficiency differs significantly among various $\mathrm{TiO}_{2}$ samples and the effect of calcination temperatures on the photocatalytic activity is obvious. Clearly, the photocatalytic activity is greater for $\mathrm{TiO}_{2}$ calcined at a lower temperature, reaching the maximum at $400{ }^{\circ} \mathrm{C}$ and then diminishing. After 90 minutes of photocatalysis TiB 600 and TiV600 samples thus display the RR45 dye decomposition rate of only $16 \%$ and $3 \%$, respectively. The exception is TiCh600 sample 
indicating a very high rate of dye decomposition, which is attributed to the crystallite size formed during synthesis and calcination. This is confirmed by XRD results where the crystallite size was $34 \mathrm{~nm}$ for TiCh600 and significantly smaller than those determined for samples TiB600 and TiV600 (41 and $51 \mathrm{~nm}$, respectively). These results as well as SEM micrographs suggest that the structure and morphology are of crucial importance for the photocatalytic efficiency of catalysts. ${ }^{[20,21]}$ Demonstrating that it is necessary to control the conditions of $\mathrm{TiO}_{2}$ synthesis during which its crystallites are formed. As observed from the SEM micrographs (Figure 5), the finest morphology is achieved at $400{ }^{\circ} \mathrm{C}$ and particularly well seen for TiCh400 sample. This is explained by the fact that both crystallinity and the surface area affect the photocatalytic activity of a photocatalyst since an increase in size reduces the surface area, which is a less active site (for oxidation/reduction). Calcination temperature can enhance the crystallinity of a photocatalyst and provide more sites for the recombination of the photogenerated electron-hole pairs, which is not preferable. The photocatalytic activity is simultaneously affected by crystallinity and by the surface area. It is necessary to obtain an optimum since due to the calcination process crystallinity can come in conflict with the enlargement of the surface area. ${ }^{[17]}$ Based on the above results it can be concluded that the photocatalytic activity was affected by crystallite and crystal sizes as a result of various synthesis methods and post-synthesis treatments. This is supported by a very high decomposition rate of RR45 achieved by TiCh400 and TiV400 photocatalysts, up to $98 \%$ after 60 minutes. It is assumed that modified synthesis conditions (duration of hydrolysis and temperature) have affected the crystallite growth rate due to calcination temperature. Furthermore, a variation in thus obtained structure of the photocatalysts obviously modifies the kinetics of the decomposition reactions during the photocatalysis since the kinetics depends on the concentration of $\mathrm{H} \bullet$ and $\mathrm{OH} \bullet$ radicals formed upon UV irradiation. Moreover, as known from literature, ${ }^{[35]}$ the $\mathrm{TiO}_{2}$ structure also has an effect on electron-hole charge carriers $\left(h^{+} V B+e^{-} C B\right)$ that can undergo recombination and dissipate excess energy through nonradiative mechanisms, thus reducing the overall efficiency of the photoinduced process.

Figure 7 displays the results of RR45 dye removal during the adsorption and photocatalytic processes involving TiB400, TiCh400 or TiV400 compared with commercial $\mathrm{TiO}_{2}$ (P25). It is evident that synthesized $\mathrm{TiO}_{2}$ photocatalyst samples present lower adsorption and slightly lower decomposition kinetics during photocatalysis in the first 60 minutes. After 90 minutes of photocatalysis all of the photocatalysts decomposed $98 \%$ of the dye.

TOC values (Figure 8) describe the extent of mineralization of an organic compound, i.e., indicate the concentration of remaining compounds or its degradation products, and all of this is expressed via the carbon atoms content, except $\mathrm{CO}_{2}$. Here TOC was used as a measure of the decomposition of RR45 molecules in water. Clearly, commercial $\mathrm{TiO}_{2}$ (P25) showed a significantly higher mineralization rate of RR45 as it decomposed almost $60 \%$ of the dye and its decomposition products in water, whereas synthesized TiV400 samples with higher TOC values decomposed about half of that ( $30 \%)$. Furthermore, the TOC values also show that the catalysts TiCh400 and TiV400 which equally efficiently decomposed the chromophore group of RR45 dye as the commercial $\mathrm{TiO}_{2}$ (Figure 7) were less efficient in the case of mineralization of RR45 dye.

The efficiency of the photocatalytic activity of synthesized $\mathrm{TiO}_{2}$ photocatalysts calcined at $400{ }^{\circ} \mathrm{C}$, most efficient in the photocatalysis of RR45 dye, was also tested for photocatalysts of Acid Blue (AB25) dye; the results are presented in Figure 9. It can be seen that the processes of adsorption and photocatalysis differ from those involving RR45 dye degradation. During the 90 minutes of establishing the adsorption/desorption equilibrium process, sample TiB400 adsorbed about $27 \%$, commercial $\mathrm{TiO}_{2} 15 \%$ and the catalysts TiCh400 and TiV400 $9 \%$ and $1 \%$ of $A B 25$ dye, respectively. It is presumed that the high level of $A B 25$ dye adsorption is due to its negatively charged sulphonate groups and positively charged $\mathrm{TiO}_{2}$ particles. In some of our earlier experiments the $\mathrm{pH}_{\mathrm{PzC}}$ of $\mathrm{TiO}_{2}$ was established at 6.6. Such a $\mathrm{pH}_{\mathrm{PzC}}$ value of $\mathrm{TiO}_{2}$ is in accordance with literature findings. ${ }^{[36]}$ The $\mathrm{pH}$ value in wastewater with RR45 in the photocatalytic experiment was around 6.2 whereas in wastewater with $A B 25$ it was around 5.5. It is known from literature that when $\mathrm{pH}$ is below the $\mathrm{TiO}_{2}$ nanoparticle $\mathrm{pH}_{\mathrm{Pzc}}$, nanoparticles exhibit a positively charged surface. ${ }^{[37]}$

Electrostatic interactions here favour dye adsorption onto the catalyst surface, thus enabling efficient photocatalytic degradation of the dye. ${ }^{[38]}$ The best photocatalytic efficiency and the highest rate of AB25 dye decomposition was obtained with a TiB400 catalyst, which decomposed 64 $\%$ of the dye in 30 minutes - slightly better than the commercial $\mathrm{TiO}_{2}$. After $90 \mathrm{~min}$. TiB400 decomposed $92 \%$ of the dye. On the other hand, samples TiV400 and TiCh400 exhibit a significantly lower rate and efficiency of AB25 dye degradation than for RR45 dye (just the opposite behaviour is observed). The photocatalysts TiV400 and TiCh400 decomposed only $28 \%$ and $47 \%$ of AB25 dye in $30 \mathrm{~min}$, and after 90 min about $83 \%$ and $85 \%$, respectively. A particularly high rate of $\mathrm{AB} 25$ decomposition by the TiB photocatalyst is explained by its smaller obtained crystallites (as estimated using the Scherrer equation) ${ }^{[18]}$ and supported by the adsorption process of $A B 25$ onto the TiB400 surface, Figure 9. 


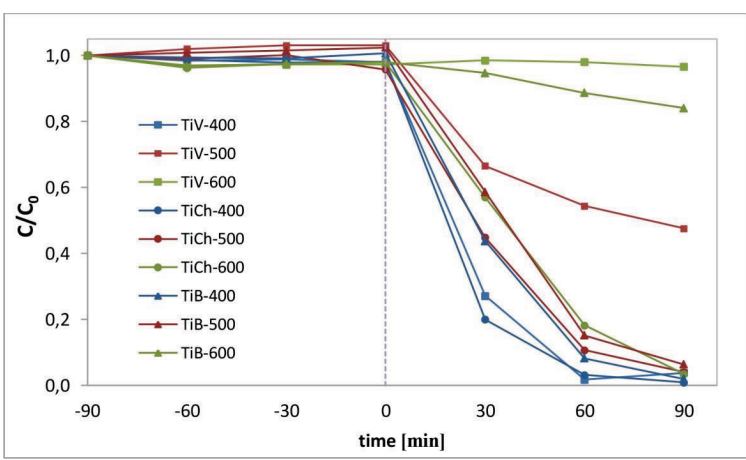

Figure 6. Concentration of removed RR45 dye during the adsorption and photocatalysis processes under UVA irradiation with synthesized $\mathrm{TiO}_{2} ; \mathrm{TiB}, \mathrm{TiCh}$, TiV calcined at $400{ }^{\circ} \mathrm{C}, 500{ }^{\circ} \mathrm{C}, 600{ }^{\circ} \mathrm{C}$ (concentration of dye RR45 $\gamma=$ $30 \mathrm{mg} \mathrm{L}^{-1}$ and photocatalyst $\gamma=1 \mathrm{~g} \mathrm{~L}^{-1}, \mathrm{pH}$ 7). Negative time denotes the part of the experiment of adsorption / desorption equilibrium before irradiation.

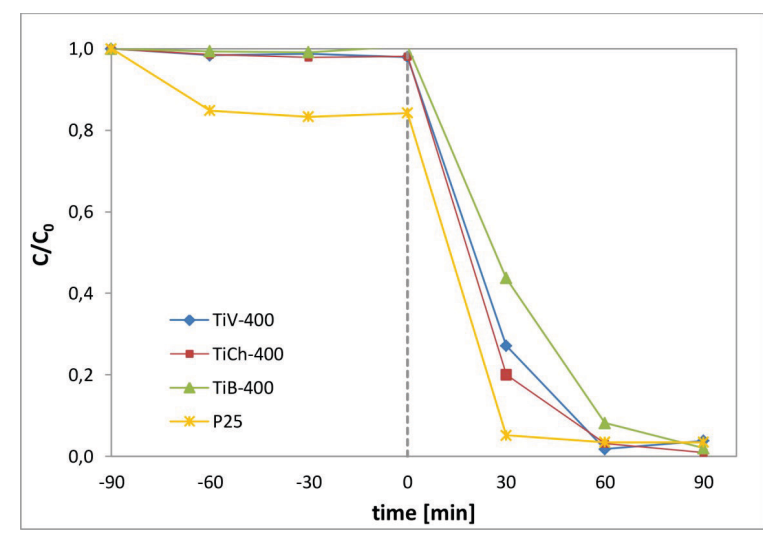

Figure 7. Concentration of removed RR45 dye during the adsorption and photocatalysis processes under UVA irradiation with synthesized $\mathrm{TiO}_{2} ; \mathrm{TiB}, \mathrm{TiCh}$, TiV calcined at $400{ }^{\circ} \mathrm{C}$ and with commercial $\mathrm{TiO}_{2}$ (P25) (concentration of dye RR45 $\gamma=30 \mathrm{mg} \mathrm{L}^{-1}$ and photocatalyst $\gamma=1 \mathrm{~g} \mathrm{~L}^{-1}, \mathrm{pH}$ 7). Negative time denotes the part of the experiment of adsorption/desorption equilibrium before irradiation.

Figure 10 shows the results of $A B 25$ dye mineralization during photocatalysis displayed as a percentage of TOC values. It can be seen that the rate of AB25 dye molecule decomposition is significantly higher than for RR45 dye since the TOC removal values are higher. This can be explained by an increased interaction between the synthesized $\mathrm{TiO}_{2}$ catalysts and $\mathrm{AB} 25$ dye as it is obvious from the adsorption process. The adsorption of the dye on the surface may enhance the photocatalytic degradation of the pollutants. In that way the kinetic rate of degradation can be accelerated resulting in higher decomposition and mineralization. As already seen from the obtained results, the

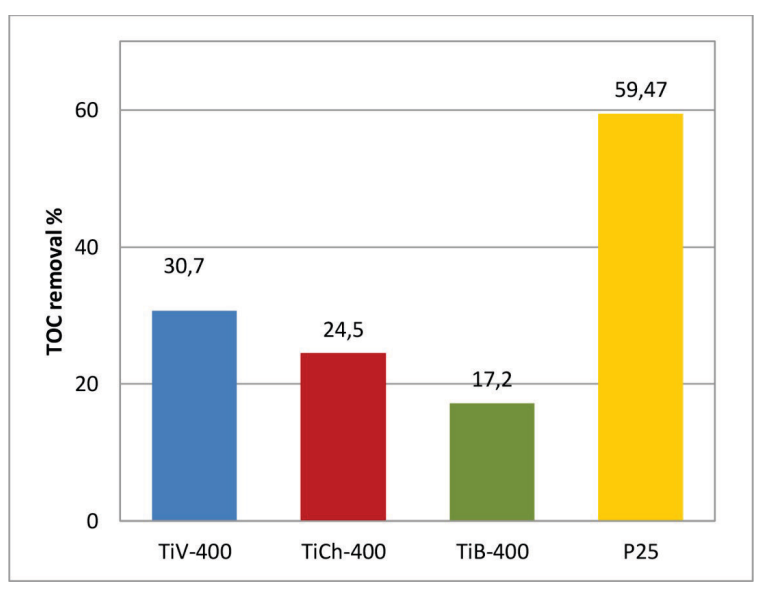

Figure 8. Percentage of TOC removal of RR45 dye during the photocatalytic degradation with synthesized $\mathrm{TiO}_{2} ; \mathrm{TiB}, \mathrm{TiCh}$, $\mathrm{TiV}$ calcined at $400^{\circ} \mathrm{C}$ and commercial $\mathrm{TiO}_{2}(\mathrm{P} 25)$.

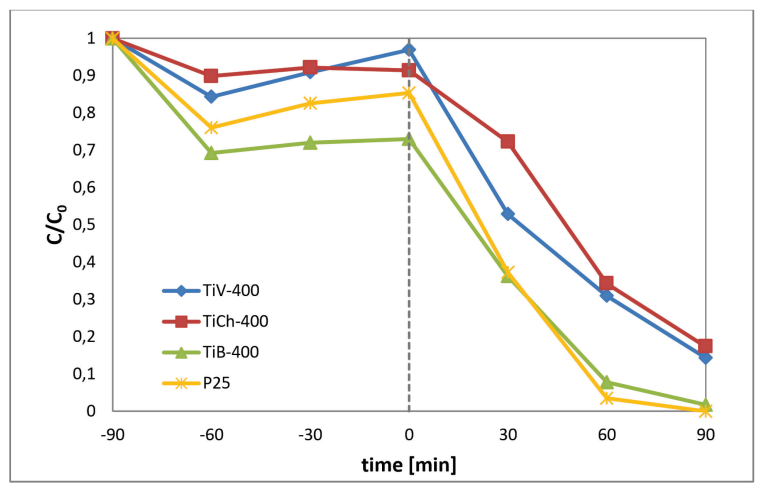

Figure 9. Concentration of removed AB25 dye during the adsorption and photocatalysis processes under UVA irradioatin with synthesized $\mathrm{TiO} 2 ; \mathrm{TiB}, \mathrm{TiCh}$, TiV calcined at $400{ }^{\circ} \mathrm{C}$ and with commercial $\mathrm{TiO}_{2}$ (P25) (concentration of dye RR45 $\gamma=30 \mathrm{mg} \mathrm{L}^{-1}$ and photocatalyst $\gamma=1 \mathrm{~g} \mathrm{~L}^{-1}, \mathrm{pH}$ 7) Negative time denotes the part of the experiment of adsorption/desorption equilibrium before irradiation.

degradation rate of dyes can be affected by crystals and crystallite size, interactions between dye and catalyst due to charge, and parameters such as $\mathrm{pH}$, catalyst concentration, pollutant concentration and the presence of electron acceptors such as molecular oxygen. ${ }^{[39]}$ Furthermore, it can be concluded that the TiB400 catalyst is more successful in the decomposition of $\mathrm{AB} 25$ dye, which is an anthraquinone type of dye with the $\mathrm{C}=0$ group, unlike RR45 that is an azo dye with the $\mathrm{N}=\mathrm{N}$ chromophore group. Vice versa, TiV400 and TiCh400 catalysts are more efficient in the degradation of RR45 azo dye. When we consider the decomposition of complete dye molecules and their formed end-products regarding the TOC values, it is evident that synthesized $\mathrm{TiO}_{2}$ 
catalysts are more effective in AB25 dye decomposition whereas the commercial $\mathrm{TiO}_{2}$ catalyst is more effective in RR45 dye decomposition. This further suggests that, in addition to photocatalytic process conditions $(\mathrm{pH}$, concentration of catalyst, concentration of pollution), ${ }^{[40,41]}$ the structure of $\mathrm{TiO}_{2}$ catalyst is very important as well as the structure and chemical composition of the pollutant.

Here it should be pointed out that, in addition to the structure (crystallinity) of a photocatalyst, its chemical composition is also important. It is supposed that $\mathrm{TiO}_{2}$ samples (Figure 2), which were synthesized by various methods, slightly differ in their chemical composition. It is assumed that there are remaining starting precursors in their structure. For further clarification elemental analysis should be carried out by some additional experiments. Namely, such remaining groups in $\mathrm{TiO}_{2}$, although present in very low concentrations, affect the interactions between the catalyst and the pollutant. The interactions are very important as they enable the adsorption of the pollutant on the catalyst surface and the photocatalysis is thus initiated.

\section{Consecutive Cycles of Photocatalysis}

The photocatalytic efficiency of synthesized $\mathrm{TiO}_{2}$ photocatalysts during several consecutive cycles of photocatalysis was determined under exactly the same conditions as in the first cycle. After each cycle photocatalysts were not additionally treated or recovered, suggesting that in each new cycle the catalysts were partly polluted with dye from the former cycle. The obtained results give the information about photocatalyst stability and its capacity of multiple use without any further costs. The percentage of degraded RR45 dye in wastewater after 90 minutes of photocatalysis during four consecutive cycles is given in Figure 11. From the results it is observed that the most stable photocatalyst during all four cycles was TiCh400, as its efficiency

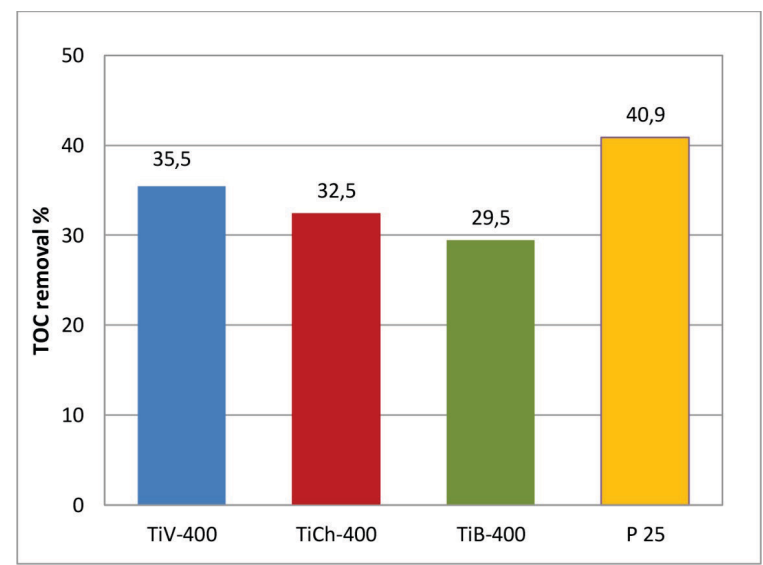

Figure 10. Percentage of TOC removal of $A B 25$ dye during the photocatalytic degradation with synthesized TiO2; $\mathrm{TiB}$, TiCh, TiV calcined at $400{ }^{\circ} \mathrm{C}$ and commercial $\mathrm{TiO}_{2}$ (P25). decreased for only $19 \%$. The same sample has shown the highest decomposition rate of RR45 (Figure 7), indicating a high stability and photocatalytic efficiency on reuse. TiB400 photocatalyst sample demonstrated the lowest photocatalytic activity after each cycle and the percentage of degraded RR45 dye was $67 \%$ after the fourth cycle. The TOC values were determined after each consecutive cycle of photocatalysis and the results are given in Figure 12. It is obvious that TOC values decrease more and more with each cycle for all synthesized $\mathrm{TiO}_{2}$ photocatalysts. Photocatalytic activity and degradation efficiency were significantly lower for TiB400 catalyst sample, amounting to just $2.6 \%$ in the fourth cycle. It can be concluded that TiB400 is not a satisfying photocatalyst for the removal of RR45 dye from water as its mineralization rate in the first cycle was

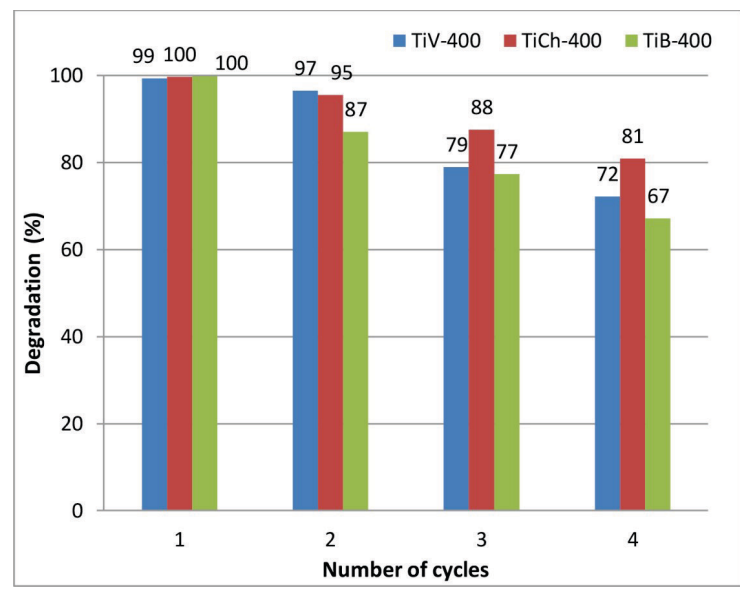

Figure 11. Degradation rate of RR45 dye after 90 minutes of photocatalysis during four cycles using TiB400, TiCh400, TiV400 photocatalysts.

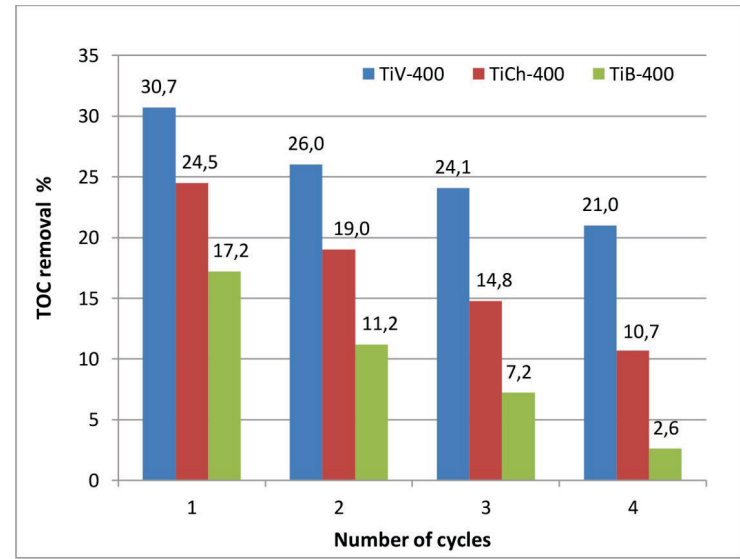

Figure 12. Percentage of TOC removal of RR45 dye after 90 minutes of photocatalysis during four cycles using TiB400, TiCh400, TiV400 photocatalysts. 
low (17.2\%). TiV400 catalyst showed a significantly higher efficiency with the highest rate of mineralization and with a TOC removal of about $30 \%$.

\section{CONCLUSIONS}

From the results of photocatalytic activity obtained with synthesized $\mathrm{TiO}_{2}$ catalysts it is well seen that the conditions of synthesis significantly contribute to the $\mathrm{TiO}_{2}$ photocatalytic efficiency. $\mathrm{TiO}_{2}$ synthesis results in the formation of various crystal structures and different sizes of crystallites. The photocatalytic process always depends on the type of the pollutant and the type of the catalyst. Due to this it is concluded that the photocatalytic degradation of RR45 azo dye is most efficient with TiCh400 due to the lower size of $\mathrm{TiO}_{2}$ crystallites. In this way the surface of the catalyst is enlarged and reveals more active sites resulting in an increased photocatalytic efficiency. Due to the different crystal structure in comparison with other samples, TiV500 (presence of anatase and rutile) also exhibits good photocatalytic activity.

Another parameter like the chemical composition of the catalyst was revealed as dominant in the photocatalytic degradation of AB25 anthraquinone dye with the TiB400 catalyst. As observed in the FTIR spectra, there are small differences in the chemical composition of the synthesized $\mathrm{TiO}_{2}$ catalysts due to the use of various starting chemicals in synthesis that were incorporated into the $\mathrm{TiO}_{2}$ structure in a very low concentration. The remaining groups of DDAC in TiB sample increased the interaction between $A B 25$ dye and the catalyst, confirmed by the highest adsorption of dye on the catalyst surface and the highest rate of degradation. A significant contribution to the increased chemical interaction between the catalyst and dye in this particular process is due to the chemical structure of dye itself. This statement is also confirmed by comparing the results of photocatalysis performed by the commercial $\mathrm{TiO}_{2}$ (P25) catalyst for RR45 and AB25. From the results it is concluded that, for the successful and economically feasible process of wastewater treatment, a universal $\mathrm{TiO}_{2}$ catalyst is not sufficiently effective.

Acknowledgment. This work was financially supported by the Croatian Science Foundation (HRZZ) within the project: Development of Photocatalytic Polymer Nanocomposites for Wastewater Treatment (5092).

\section{REFERENCES}

[1] K. M. G. Mostofa, Y. Honda, H. Sakugawa, Geochem. J. 2005, 39, 257.

[2] N. Mirzoyan, Y. Tal, A. Gross, Aquaculture 2010, 306, 1.

[3] M. Saleem, M.H. Essa, Univ. J. Res. 2010, 7, 23.
[4] J. Rubio, J. L. Oteo, M. Villegas, P. Duran, J. Mater. Sci. 1997, 32, 643.

[5] S. Hu, A. Wang, X. Li, H. Lowe, J. Phys. and Chem. of Solids 2010, 71, 156.

[6] M. Matsuoka, M. Anpo, Photobiol. C: Photochem. Rev. 2003, 3, 225.

[7] M. Anpo, H. Yamashita, Y. Ichihashi, S. Ehara, J. Electroanal. Chem. 1995, 396, 21.

[8] M. Anpo, J. M. Thomas, Chem. Commun. 2006, 31, 3273.

[9] Y. Horiuchi, T. Toyao, M. Takeuchi, M. Matsuoka, M. Anpo, Phys. Chem. Chem. Phys. 2013, 15, 13243.

[10] K. Mori, Y Miura, S. Shironita, H. Yamashita, Langmuir 2009, 25, 11180.

[11] H. Yamashita, K. Mori, Chem. Lett. 2007, 36, 348.

[12] H. Yamashita, K. Mori, S. Shironita, Y. Horiuchi, Catal. Surv. Asia 2008, 12, 88.

[13] D. R Kennedy, M. Ritchie, J. Mackenzie, J. Trans. Faraday Soc. 1958, 54, 119.

[14] Y. Fujita, T. Kwan, Bull. Chem. Soc. Jpn. 1958, 31, 379.

[15] W. Doerffler, K. Hauffe, J. Catal. 1964, 3, 156.

[16] J. Schneider, M. Matsuoka, M. Takeuchi, J. Zhang, Y. Horiuchi, M. Anpo, D. W. Bahnemann, Chem. Rev. 2014, 114, 9919.

[17] Q. Chena, W. Shia, Y. Xua, D. Wua, Y. Suna, Mater. Chem. Phys. 2011, 125, 825.

[18] H. P. Klug, L. E. Alexander, X-Ray diffraction procedures for polycrystalline and amorphous materials, 2nd Ed., Wiley, London, 1974, p. 81.

[19] N. Sobana, M. Muruganadham, M. Swaminathan, J. Mol. Catal. A: Chem. 2006, 258, 124.

[20] H. G. Yang, C. H. Sun, S. Z. Qiao, J. Zou, G. Liu, S. C. Smith, H. M. Cheng, G. Q. Lu, Nature 2008, 453, 638.

[21] Z. S. Wang, H. Kawauchi, T. Kashima, H. Arakawa, Coord. Chem. Rev. 2004, 248, 1381.

[22] Y. Ma, X. Wang, C. Li, Chem. Rev. 2014, 114, 9987.

[23] P. Kormali, A. Troupis, T. Triantis, A. Hiskia, E. Papaconstantinou, Catal. Today 2007, 124, 149.

[24] Q. Chena, W.Shia, Y. Xua, D. Wua, Y. Sun, Mater. Chem. Phys. 2011, 125, 825.

[25] M. A. Henderson, Surf. Sci. Rep. 2011, 66, 185.

[26] A. L. Linsebigler, G. Lu, J. T.Yates, Chem. Rev. 1995, 95, 735.

[27] Q. Sheng, S. Yuan, J. Zhang, F. Chen, Microporous Mesoporous Mater. 2006, 87, 177.

[28] R. López, R. Gómez, S. Oros, Catal. Today 2011, 166, 159.

[29] L. Dong, M. Li, L. Dong, M. Zhao, J. Feng, Y. Han, J. Deng, X. Li, D. Li, X. Sun, Int. J. Hydrogen Energy 2014, 39, 16116.

[30] Y. Djaoued, S. Badilescu, P.V. Ashrit, D. Bersani, P.P. Lottici, J. Robichaud, J. Sol-Gel Sci. Techn. 2002, 24, 255. 
[31] H. P. Klug, L. E. Alexander, X-ray diffraction procedures for polycrystalline and amorphous materials, 2nd Ed., John Wiley \& Sons, New York, 1974, p. 112.

[32] B. Haspulat, A. Gülce, H. Gülce, J. Hazard. Mater. 2013, 260, 518.

[33] Q. Guo, C. Zhou, Z. Ma, Z. Ren, H. Fan, X. Yang, in Heterogeneous Photocatalysis: From fundamentals to green applications (Eds. Y.J. Xu, J.C. Colmenares), Springer-Verlag, Berlin, Heidelberg, 2016, p. 361.

[34] K. Y. Jung, S.B. Park, J. Photochem. Photobiol. A: Chem. 1999, 127, 117.

[35] S. Banerjee, S. C. Pillai, P. Falaras, K. E. O'Shea, J. A. Byrne, D. D. Dionysiou, J. Phys. Chem. Lett. 2014, 5, 2543.
[36] N. T. Boncagni, J. M. Otaegui, E. Warner, T. Curran, J. Ren, M. M. Fidalgo de Cortalezzi, Environ. Sci. Technol. 2009, 43, 7699.

[37] F. Loosli, P. Le Coustumer, S. Stolla, Water Res. 2013, 47, 6052

[38] M. Luo, D. Bowden, P. Brimblecombe, Water Air Soil Pollut. 2009, 198, 233.

[39] I. K. Konstantinou, T. A. Albanis, Appl. Catal. B 2004, $49,1$.

[40] N. Daneshvar, D. Salari, A. R. Khataee, J. Photochem. Photobiol. A 2003, 157, 111.

[41] N. Daneshvar, D. Salari, A.R. Khataee, J. Photochem. Photobiol. A 2004, 162, 317. 